\title{
Efficient Image Restoration Methods for Image Recovery
}

\author{
G. Kalpana, A. Kanaka Durga, G.Karuna
}

\begin{abstract}
Image Restoration is the one of the significant techniques to process an image. The process of taking a noisy image and obtaining the clean, original image is called as image restoration. This process is applied in every field where images have to be understood and analyzed. Image restoration is a method of recovering an original image from a degraded image. To restore corrupted image into its original form restoration techniques are used. The restoration techniques mainly focused to improve the image quality. Usually image processing techniques are implemented in the two domains, they are frequency domain or spatial domain. This paper mainly focused on different approaches to restoration, variations between frequency domain methods and spatial domain methods. Especially the relation between spatial and frequency resolutions and various filters in spatial and frequency domain. The present work shows the performance of different kinds of filters and these filters are analyzed by implementing and simulating on MATLAB.
\end{abstract}

Keywords : Image restoration, filters, spatial Domain, frequency Domain

\section{INTRODUCTION}

Digital images play an important role in the fields, such as remote sensing, television, ultrasound, Biomedical imaging like CT scan etc[1-3]. Image Processing is a method of processing an original images captured from cameras/sensors placed on artificial satellites, military and aircrafts or the images taken from real life for different applications[4]. Image can be converted in to digital form to perform operations on it like image acquisition, image enhancement, segmentation, image restoration, image compression, transformation etc.

To remove or reduce noise (motion blur, sensor noise etc..) from the image , Image restoration or denoising is necessary. To analyze and interpret most important information of an image, noise reduction is a fundamental operation of image processing. Two categories of techniques are widely used to perform restoration i.e under spatial domain and under frequency domain. This paper focuses brief general classification of few denoising methods under spatial and frequency domains [5].
Here $f(x, y)$ is the original input image, $n(x, y)$ is the noise to be added, $g(x, y)$ is the image after additive noise to original input image and $\mathrm{f}^{1}(\mathrm{x}, \mathrm{y})$ is the processed output image after applying the restoration filtering techniques.

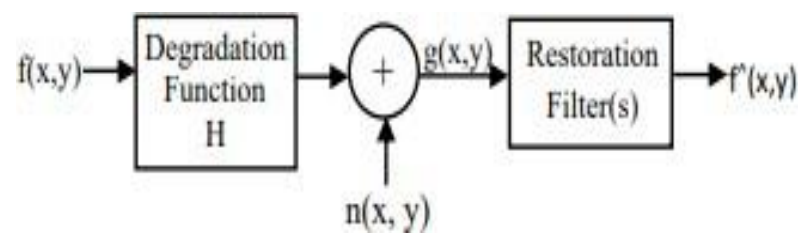

Fig1: Image Restoration process [3]

$$
\text { original Image }
$$

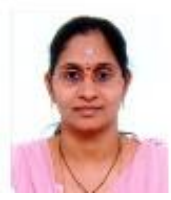

Fig2: Original Color Image gray image

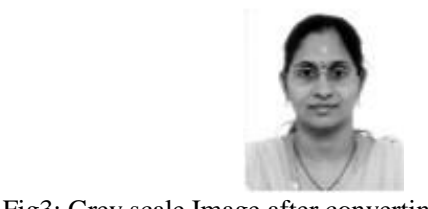

Fig3: Grey scale Image after converting from color image

\section{SPATIAL DOMAIN}

In this domain, various operations can be performed directly on images. A digital image is a grid of pixels. The smallest element of an image is a pel or pixel. Each pixel represents to any one value called pixel intensity or strength. The intensity of an image is in variance with the location of a pixel.

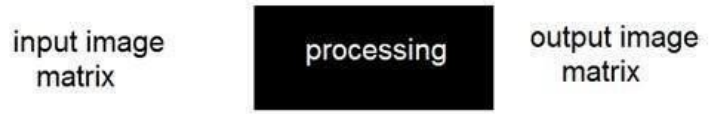

Fig4: Spatial Domain

Let I be an image and (x,y)is the location (or coordinate) of any pixel and the image is depicted as a function of location : $\mathrm{I}(\mathrm{x}, \mathrm{y})$ where $\mathrm{x}$ and $\mathrm{y}$ are integers. So an image $\mathrm{I}(\mathrm{x}, \mathrm{y})$ is a matrix of pixels. 
The term spatial refers to space. In an image, this space is a 2D plane (xy-plane).The spatial domain represents the image plane itself and various approaches in spatial domain are based on directly modifying the value of the elements or pels. Spatial domain processes are represented as

$$
\mathrm{I} 1(\mathrm{x}, \mathrm{y})=\mathrm{T}[\mathrm{I}(\mathrm{x}, \mathrm{y})]
$$

Where I1 is improved image and the pixel or pel value with coordinates $(\mathrm{x}, \mathrm{y})$ in $\mathrm{I} 1$ is the result of performing some operation $\mathrm{T}$ on the pixels in the neighborhood of $(\mathrm{x}, \mathrm{y})$ in the original image I.[6]

\section{FREQUENCY DOMAIN}

In frequency domain an image is first transformed frequency distribution. Then the black box system perform its necessary functions. The output of the black box is a conversion only and not an image. After the process of inverse transformation is over, it is converted into an image which is then viewed in spatial domain. It can be pictorially viewed in brief as

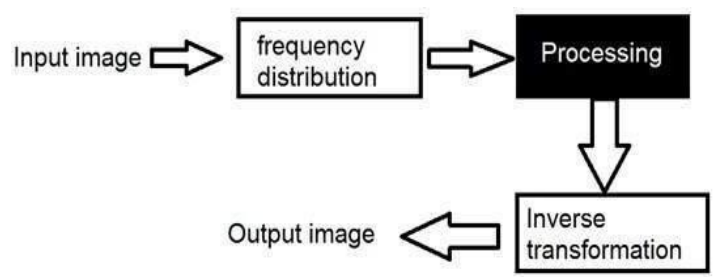

Fig5: Frequency Domain

There are mainly 3 important major steps to perform frequency domain filtering [7]. They are, 1 . The image must be transformed from the spatial domain into the frequency domain using the Fourier transform. 2. The resulting complex image must be multiplied by a filter.

$$
f(x, y) h(x, y) \Leftrightarrow H(u, v)^{*} G(u, v)
$$

3 . The filtered image must be transformed back in to the spatial domain.

Frequency components are mainly divided into high frequency and low frequency components. High frequency components are correspond to edges in an image. Whereas the low frequency components are correspond to smooth regions of an image.[7]

Using mathematical operators a signal can be converted from time domain into frequency domain called transforms. There are many types of transformation that does this. Some of them are a) Fourier Series b) Fourier transformation c) Laplace transform d) $\mathrm{Z}$ transform. The Fourier Transform is most widely utilizing image processing approach that is used to decompose an image into two components ie sine and cosine. The output of that transformation represents the image in the Fourier or frequency domain, but the input image is the spatial domain equivalent. Traditional Fourier transform algorithm computes the value of each point of image, regardless of their properties in the frequency domain $[8,12]$.

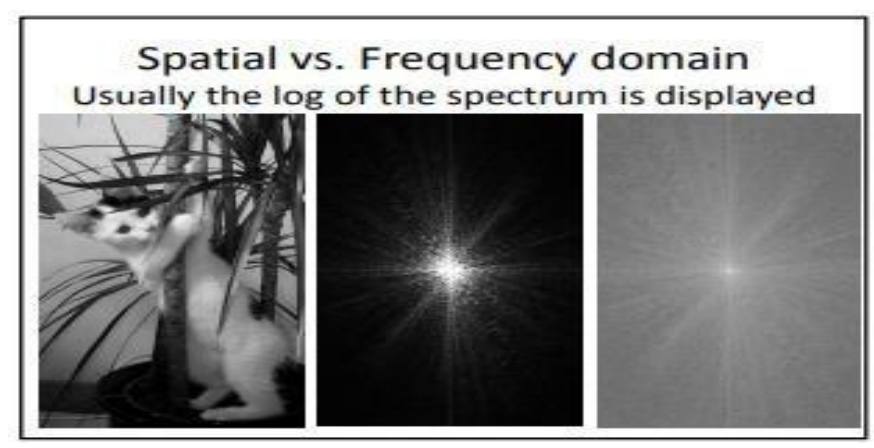

Fig6: Spatial Vs Frequency Domain

\section{THE RELATION BETWEEN SPATIAL DOMAIN AND FREQUENCY DOMAIN}

A. Relation Between Spatial and Frequency Resolutions:

$\Delta u=\frac{1}{M \Delta x}$ and $\Delta v=\frac{1}{N \Delta y}$

Where $\Delta x$ and $\Delta y$ are pixel width and height

- $\Delta x$ is spatial resolution in $\mathrm{x}$ direction

- $\Delta y$ is spatial resolution in y direction

- $\Delta u$ is frequency resolution in $\mathrm{x}$ direction

$-\Delta v$ is frequency resolution in $\mathrm{y}$

direction Where $\mathrm{N}, \mathrm{M}$ are Image width and height

\section{B. Discrete Fourier Transform relate to Spatial}

\section{Domain Filtering}

Following convolution theorem shows an interesting relationship between spatial domain and frequency domain:

[where $h(x, y)$ filter mask, $H(u, v)$ is a filter function, $\mathrm{F}(\mathrm{u}, \mathrm{v})$ is DFT of input image]

and, conversely,

Here symbol "*" indicates convolution of two functions. Extracting an image out of this is that the multiplication of two Fourier transforms corresponds to the convolution of the associated functions in spatial domain.

\section{FILTERS IN SPATIAL AND FREQUENCY DOMAIN}

In the spatial domain digital image filtering means modifying the pixels in an image based on a function on a local neighborhood

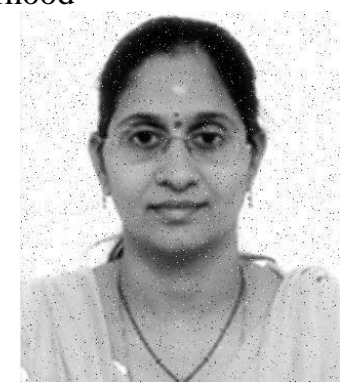

Fig 7: Image with Salt and pepper noise 


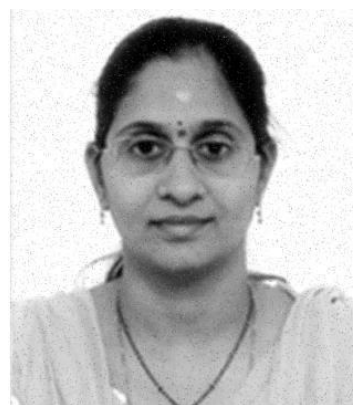

Fig 8: Image with average Filter

The Average Filter is simplest approach to reducing noise and it is implemented by replacing every pixel value with the average of neighborhood around it. The noise amplitude is reduced by a factor equal to the square root of the number of pixels involved in the smoothing.

In Order Statistic Filter, the values of the pixels of an image are ranked in order. Those pixels of image are ranked whose area or region is enclosed in the filter.

In Median Filter the median of the intensity levels of the pixels are first calculated. For example if the pixel values are from 1 to 9 , then the median will be 5 , that is, the midpoint of the pixel values. The new value is replaced with the corrupted pixel value after calculating the median. It never affects median value because single pixel in the neighborhood .so this filter is more robust. To preserve sharp edges this filter is much better than any other filter.

But To execute it is more expensive and complex. For every window it will take large amount of time to calculate a median value [9-11].

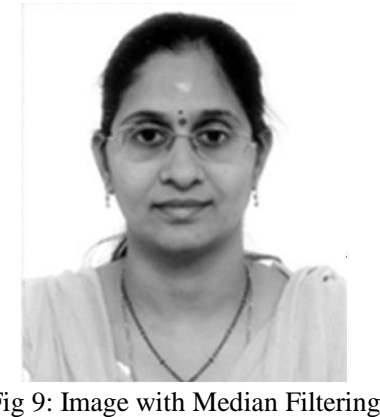

To find the brightest and darkest locations in the image usually Max and Min Filters are used. The Min filter replaces the pixel with the darkest point and Max filter substitutes the pixel or pel value with the brightest point. Max filter finds light colored pixels in an image while Min filter finds dark points in the image.

\section{ORIGINAL IMAGE}

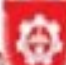

Vidya Jyothi

Institute of Technology

Fig 10: Original Image
IMAGE AFTER MIN FILTERING

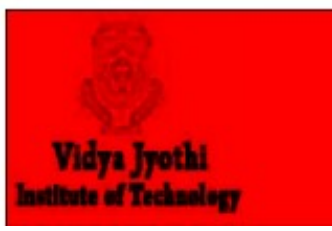

FIG 11: IMAGE WITH MIN FILTERING

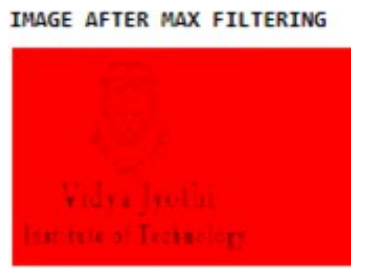

FIG12: IMAGE WITH MAX FILTERING

Midpoint Filter is calculated midpoint by taking middle value between the maximum and minimum values of the image. It is widely used for noises like Gaussian noise and uniform noise. but for only Alpha-trimmed Mean Filter trims the $\mathrm{d} / 2$ highest and $\mathrm{d} / 2$ lowest intensity values of the corrupted image in Sxy. Let $\operatorname{gr}(\mathrm{s}, \mathrm{t})$ represents remaining mn-d pixels. The alpha trimmed mean filter caluculates averages of the value of these remaining pixels. ie $\mathrm{d}$ ranges from 0 to $\mathrm{mn}-1$. Alphatrimmed filter becomes arithmetic mean filter, when $\mathrm{d}=0$. The filter becomes a median filter, if $d=m n-1$. In multiple categories of noise such as Gaussian noise, and the combination of salt and pepper noise it is useful.

This Adaptive Mean Filter is a third type of spatial domain filters. The filter size will be changed, in adaptive mean filter. For the images where the density of the noise is less, the other filters discussed above can only be used. But to remove high-density noise from corrupted images this filter is especially used [9-11].

The digital image filtering is considered in the frequency domain which is a process of discriminating from undesired frequency components to desired frequency components.

Low pass filters are applied for image smoothing and high pass filters are used for getting the sharp edges of the image. These filters remove the unwanted frequency components in the frequency domain. The removal of high frequency results in image smoothing and noise reduction [9-10].

High pass filters also operate in frequency domain which remove low frequency elements of frequency domain and result in a sharper image where the edges in the images or the sudden change if intensities are preserved. This helps a lot in edge detection [9-10]. 


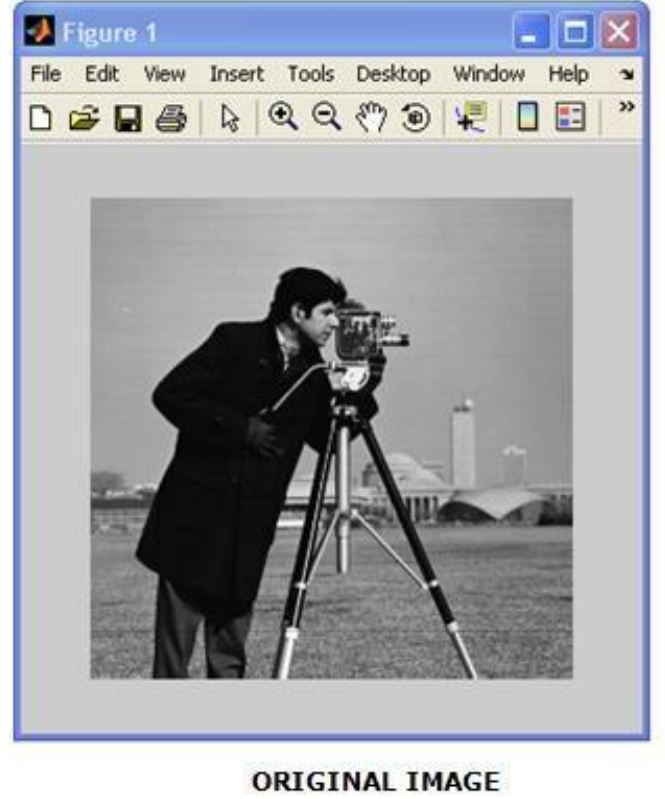

Fig13: Original Image

\section{RESULT ANALYSIS AND DISCUSSIONS}

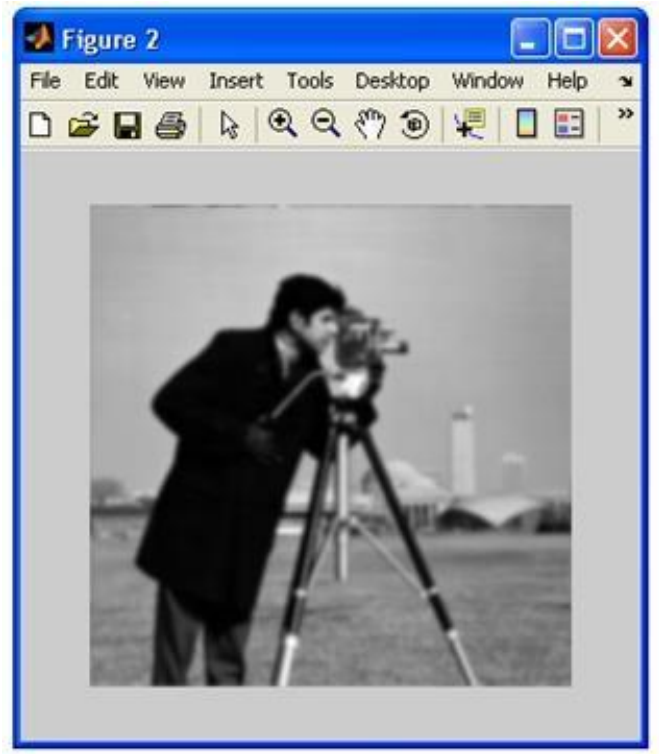

IMAGE AFTER Gaussian LPF

fig 14: Image after Gaussian LPF

The present work taken nearly 20 sample images with various dimension and we have applied few filtering techniques to process and restore the original image. This work mainly taken simulated and observed results as follows. First we have taken an input original color image(fig 2) and converted into gray scale image(fig 3) and The image is first exposed to a salt-and-pepper noise (fig 7) and then applied Average filter it removes few noise(fig 8).finally applied median filtering technique to remove the noise completely. The resulting image is noise free( fig 10) has a better view and, as can be seen, the edges of the figure are not removed. And also we have taken another original image (fig 10) first we applied min filtering technique it replaces pixels with darkest points and makes the image more thicker (fig 11). And then we have applied max filter on the image it substitutes the pixel value with brightest points and changes the image as more brighter (fig 12). We have taken another different image (fig 13) and applied Gaussian low pass filter (14) it results in smoothing and noise reduction and it is faster than median filter

In both spatial and frequency domains similar jobs can be done. In spatial domain, images are dealt as it is. The pixel values of the image change with respect to scene. But in frequency domain, we deal with the rate at which the pixel values are changing in spatial domain. Frequency domain analysis is a very important part in image processing, especially in image enhancement, noise reduction and image filtering.

Filtering in spatial domain can be easier to understand and it also best method for contrast enhancement. For removing random noise spatial domain techniques are particularly useful. It makes more meaningful to filter in the spatial domain using small filter mask.

The frequency domain filtering approach is faster especially in larger images. It is more computationally efficient to do the filtering in frequency domain. Frequency domain techniques are useful for removing periodic noise also best method for image sharpening [9].

\section{CONCLUSION}

This paper summarized the image restoration approaches and few filtering techniques under spatial and frequency domains. Spatial domain provides high Spatial resolution and easy to perform. Filtering in the frequency domain have shown greater performance especially in larger images. This work gives great experience to understand differences among various filtering techniques through keen analysis of their respective results. These two spatial and frequency domain methods are both important and applicable in different technologies. This paper presents a comparison between both approaches along with results and also shown their pros and cons. This paper helps the authors who wants to develop a new restoration technique under spatial and frequency domains.

\section{REFERENCES}

1. Madhuri Khatri, Raunakraj ,"A Survey on Image Denoising methods"

2. Monika Maru ,M. C. Parikh ".Image Restoration Techniques: A Survey

3. Inderpreet Singh, Nirvair Neeru Paper Titled "Performance Comparison Of Various Image Denoising Filters Under Spatial Domain", International Journal of Computer Applications, (0975 8887), Volume 96-No.19, June 2014.

4. G.Kalpana,G.Karuna, "Orthogonal wavelet Decomposition With Multi-Level Thresholding For Image Enhancement”,Ijar,Int. J. Adv. Res. 5(4), 1967-1774 , Issn: 2320-5407

5. Madhuri Khatri, Raunakraj "A Survey on Image Denoising methods

6. G.Kalpana "A Comparative Study Of Image Restoration Filters Under Spatial Domain,IJCRT,Volume 6,Issue 2, April 2018,ISSN:2320-2882

7. Swati Dewangan, Anup Kumar Sharma" Image Smoothening and Sharpening using Frequency Domain Filtering Technique", International Journal of Emerging Technologies in Engineering Research (IJETER) Volume 5, Issue 4, April (2017)

8. Sheng Shi ; Runkai Yang ; Haihang You "A new two-dimensional Fourier transform algorithm based on image sparsity" 
9. "Digital Image Processing" R. C. Gonzalez and R. E. Woods, 2nd Ed., Englewood Cliffs, Nj: Prentice Hall, 2002.

10. Monika Maru ,M. C. Parikh "Image Restoration Techniques: A Survey

11. Prabhishek Singh, Raj ShreeOn "A Comparative Study To Noise Models And Image Restoration Techniques,"

12. Ashish Girdhar and Monty J Singh "Review of Transforms used in Image Enhancement" International Journal of Computer \& Mathematical Sciences IJCMS ISSN 2347 - 8527 Volume 6, Issue 12 December 2017

\section{Authors ProfiLe}

1) G.Kalpana, Asst.Professor, Dept.of C.S.E, VJIT, Hyderabad

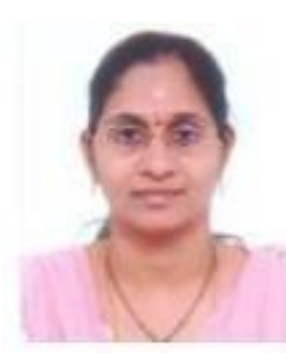

G.Kalpana is an Assistant Professor in the Department of Compute Science and Engineering, VidyaJyothi Institute Of Technology, Hyderabad, Telangana., India. She has persued her M.Tech. in CSE from HITAM college, JNTU, Hyderabad, India in 2010. She did her M.C.A from Annamalai University, Tamil Nadu, India in 2007. She has 13 years experience of

teaching for undergraduate students and for post graduate students. She has published 10 papers in various National/international journals and Conferences. Her research areas are Image Processing, Data mining and Analytics.

2) Dr. A. Kanaka Durga, Professor in CSE and HOD - IT at Stanley College of Engineering and Technology for women, Hyderabad, Telangana, India

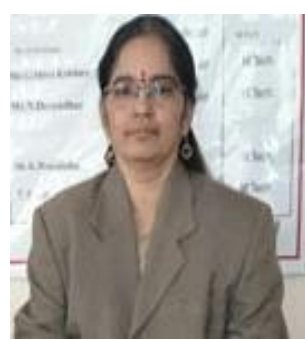

Dr.A.KanakaDurga is presently working as professor in C.S.E and HOD IT at Stanley College of Engineering and Technology for Women Heading the Department of IT of Stanley College of Engineering and technology from 2010 to till date. She has completed her M.Tech from Birla Institute of Technology, Mesra, Ranchi in the year 2001 and completed her Ph.D from JNTUH in the year 2015.She holds the responsibility of CSISSC,Rerion-V, Telangana from 2016 to till date. She was an active members in Free Software Movement in India. She has completed Govermentof AP funded project worth 5 lakhs from Ministry of IT on localization activity. She has published 19 papers in National, International conferences and Journals. Her areas of interest Data Mining, Information retrieval Systems, Machine Learning, Semantic Web, Cloud Computing, Data security, Cognitive Science, Big Data.

3) Dr.G.Karuna ,Professor, Dept of C.S.E, GRIET, Hyderabad

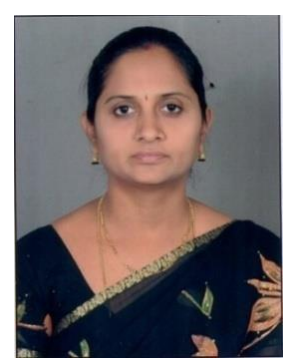

Dr.G.Karuna is presently working as a Professor in CSE Department at Gokaraju Rangaraju Institute of Engineering and Technology, Hyderabad, Telangana, India. She received her Ph.D. degree in CSE from JNTUH, Hyderabad, Telangana, India. She has thirteen years of experience in teaching for both undergraduate and post graduate students. She has a CSI, ISTE life membership. She published/presented 25 papers in various international and national journals/conferences. Her areas of research are Image Processing, Computer Networks,

Cryptography and Network Security, Big Data Analytics, Machine Learning. 